\title{
Impact of pelvic radiotherapy on female sexuality
}

\author{
Ana Cláudia Rodrigues $\cdot$ Rubina Teixeira $\cdot$ \\ Tânia Teixeira $\cdot$ Sofia Conde $\cdot$ Paula Soares $\cdot$ \\ Isabel Torgal
}

Received: 18 December 2010/ Accepted: 6 July 2011

(C) Springer-Verlag 2011

\begin{abstract}
Purpose To assess physical and psychological morbidity, sexual functioning and social and relationship satisfaction among women treated with pelvic radiotherapy.

Methods Observational (case-control) study of 199 women: 98 submitted to pelvic radiotherapy for the treatment of uterine, rectal or anal cancers and 101 without a personal history of cancer and similar socio-demographic variables. These completed a socio-demographic and clinical questionnaire, and validated measures of psychological health (DASS: Lovibond and Lovibond in Behav Res Ther 33:353-343, 1995), sexual function (FSFI: Rosen et al. in J Sex Marital Ther 26:191-208, 2007), social
\end{abstract}

\section{A. C. Rodrigues}

Department of Gynecology and Obstetrics,

Coimbra University Hospital, Coimbra, Portugal

\section{A. C. Rodrigues}

Lusophone University of Humanities and Technology,

Lisbon, Portugal

\section{A. C. Rodrigues $(\bowtie)$}

Hospitais da Universidade de Coimbra, Praceta Dr. Mota Pinto, 3000-075 Coimbra, Portugal

e-mail: anaclaudiasr@sapo.pt

R. Teixeira $\cdot$ T. Teixeira $\cdot$ S. Conde $\cdot$ P. Soares

Department of Radiotherapy, Coimbra University Hospital,

Coimbra, Portugal

I. Torgal

Department of Gynecology, Coimbra University Hospital,

Coimbra, Portugal

I. Torgal

Department of Gynecology, Faculty of Medicine,

University of Coimbra, Coimbra, Portugal support (ESSS: Ribeiro in Analise Psicologica 3:547-558, 1999) and relationship satisfaction (IMS: Hudson in The WALMYR assessment scales scoring manual 1992).

Results Women submitted to pelvic irradiation reported a higher rate of adverse physical symptoms in the last month: fatigue 59 versus $25 \%(p<0.001)$, lack of strength 42 versus $20 \%(p=0.001)$, diarrhoea 24 versus $12 \%$ $(p=0.032)$, vaginal discharge 17 versus $7 \%(p=0.024)$, skin erythema 9 versus $2 \%(p=0.026)$. Levels of depression, anxiety and stress were higher among radiotherapy patients, but only reach statistical significance for the stress parameter (6.1 vs. 4.0, $p=0.012$ ). Also these women reported lower scores of satisfaction with social support (57.2 vs. $62.2, p=0.005)$ and sexual function (8.5 vs. 13.5 , $p=0.049)$. No statistically significant differences occurred between the two groups regarding scores of relationship satisfaction (20.8 vs. $19.9, p=$ n.s.).

Conclusions Our results suggested that pelvic radiotherapy had a negative impact on female sexuality. Thus, interventions that would help to reduce this impact need to be designed and integrated into routine clinical practice.

Keywords Female sexuality - Pelvic radiotherapy · Cancer

\section{Introduction}

Cancer is a major public health problem in Portugal and in many other parts of the world [1,2]. Among the different locations of cancer, the incidence of those localized in the female pelvis is particularly high. In Europe, colorectal cancer is the second most frequent form of cancer in women $(13.25 \%)$ followed by cancer of the uterus (9.9\%) [1]. In North America, colorectal and uterine corpus 
cancers represent 10 and $6 \%$ of all incident cases in women, respectively [2].

Although cancer is the leading cause of death among women aging 40-79 years, the survival rates are increasing and consequently more attention is being paid to quality of life issues, such as sexuality [2-5].

Radiotherapy is a common therapeutic option in pelvic malignancies. However, ionizing radiation can threaten sexuality by modifying the anatomy and physiology of genital organs, interfering with the physical condition needed for sexual intercourse, leading to psychological and social changes, undermining the body image, and compromising couple intimacy [3, 6-9]. Previous research has shown that more than $50 \%$ of women treated with radiotherapy for cervical cancer experienced some form of sexual dysfunction [5, 8]. Lange et al. [9] reported dyspareunia in $59 \%$ and vaginal dryness in $57 \%$ of women after rectal cancer treatment.

Physiologic and anatomic changes that result from pelvic irradiation include impaired bowel function, urinary complaints, fatigue, pelvic pain, weight loss, skin erythema, loss of hair in the irradiated area, vaginal fibrosis causing vaginal shortness and loss of elasticity, vaginal dryness with higher risk of infection and dyspareunia, irritation in contact with the ejaculated semen, vaginal bleeding due to fragile vessels, impaired orgasm and impaired sexual arousal due to altered blood flow and denervation, infertility and premature menopause [3, 7-10]. Simultaneously, women experience symptoms of anxiety and depression, associated with the feeling of loneliness in the treatment room, the immobilisation, the uncomfortable position, the skin markings and the fear of developing skin burns, of becoming radioactive or of having new forms of cancer due to the exposure to radiation $[3,6]$.

Several studies on the topic of female sexuality after pelvic irradiation have been published. Nevertheless, the existing publications use small samples and non-validated questionnaires, do not assess all domains of sexual function and do not take into account the influence of other factors on sexuality, such as the presence of psychological morbidity and of social support or the quality of couple relationship [4, 7, 9, 11].

Within this context, the authors propose to: (1) assess physical and psychological morbidity, sexual function, satisfaction with social support and couple relationship in women with uterine, rectal or anal cancer who have received pelvic irradiation; (2) analyze how these factors evolve and relate to each other, and also how they relate to socio-demographic and clinical factors and to irradiation techniques; (3) raise the awareness of health professionals for the need to make the assessment of sexual function a part of routine care of women with cancer who receive pelvic radiotherapy, identify women with sexual problems and provide counselling.

\section{Materials and methods}

\section{Sample}

Participants were women with uterine, rectal or anal cancer, who received pelvic radiotherapy (study group) and women without cancer who attended a routine cervical cancer screening (control group). Eligibility criteria for patients were: (1) be at least 18-year old, (2) have a partner, (3) have a histological diagnosis of uterine, rectal or anal cancer, (4) have received pelvic radiotherapy for curative purposes concluded 3 months to 5 years prior to the study, (5) have a follow-up appointment between February and September 2009, (6) have no physical or psychological handicap that might interfere with questionnaire response, and (6) be able to speak and read portuguese. Eligibility criteria for controls were: (1) be at least 18-year old, (2) have a partner, (3) have no history of cancer in any location, (d) have a gynaecology appointment for routine cervical cancer screening between February and September 2009 , e) have no physical or psychological handicap that might interfere with questionnaire response, and f) be able to speak and read portuguese.

Patients were selected through the computerized cancer registry and appointment system of the Radiotherapy Unit of a tertiary care Portuguese University Hospital and controls were recruited from the Gynaecology Unit of the same University Hospital. At the appointments, the attending physician confirmed eligibility and invited all women who fulfilled the criteria for inclusion in the research to fill in a questionnaire. Women who accepted to voluntarily participate in the study were requested to give prior informed consent and filled in the questionnaire individually and without disturbance, in an appropriate room. Both the hospital's ethic committee and the administration granted their permission to conduct this study.

A total of 136 patients who were at least 18 years old, had a history of uterine, rectal or anal cancer which was treated with pelvic radiotherapy between 3 months and 5 years previously and had a follow-up appointment between February and September 2009 were identified. Of these, 3 missed the follow-up appointment, 22 were ineligible (11 for not having a partner, 9 for not being able to speak and read portuguese and 2 for having a physical or psychological handicap that might interfere with questionnaire response) and 13 declined to participate, resulting in 98 women who completed their participation in the study. 
Concerning the controls, from February to September 2009, 218 women were seen for routine cervical cancer screening. Of these, 52 did not fulfil the eligibility criteria. The most common reason for exclusion was not having a partner $(n=32)$. Among the 166 women who were eligible, 101 agreed to participate.

\section{Socio-demographic characteristics}

In the study group, the minimum age was 28 years and the maximum 85 years, with an average age of $58.2 \pm 14.8$ years; $12.3 \%$ of women were under $40,35.7 \%$ were between 41 and 60 years old and the remaining women, $52.0 \%$, were over 60 years of age. Regarding education, most women $(67.3 \%)$ had primary education and $24.5 \%$ had secondary education. As for the employment situation, $45.9 \%$ were retired, $24.5 \%$ had paid employment, $19.4 \%$ had non-paid employment (housewives and students) and $10.2 \%$ were unemployed. Moreover, $69.4 \%$ of the patients lived in rural areas. Average parity was $2.1 \pm 1.4$ children.

In the control group, age ranged from 21 to 83 years (average of $57.2 \pm 14.4$ years); $11.9 \%$ of the women were under 40, 46.5\% were between 41 and 60 years old and the remaining $41.6 \%$ were over 60 years of age. Regarding education, $62.4 \%$ of these women had primary education, $27.7 \%$ had secondary education and $9.9 \%$ had higher education. In terms of employment, most women were retired $(38.6 \%)$. Moreover, the majority (79.2\%) also lived in rural areas. Average parity was $2.1 \pm 2.1$ children.

The socio-demographic characteristics of women who received pelvic radiotherapy and of women in the control group were compared and there were no statistically significant differences between the two groups (Table 1).

\section{Clinical characteristics}

In the study group, 30 women $(30.6 \%)$ mentioned having regular menstrual cycles before beginning cancer treatment. All women were amenorrheic after treatment and $14.3 \%$ were using hormone replacement therapy. The sample of women who received pelvic radiotherapy included 48 cases $(49.0 \%)$ of cervical cancer, 34 cases (34.7\%) of endometrial cancer, 13 cases $(13.3 \%)$ of rectal cancer and 3 cases $(3.1 \%)$ of anal canal cancer. The distribution according to stage is described in Table 2. Regarding the type of treatment provided, $7.1 \%$ of women received exclusive radiotherapy, $42.9 \%$ received radiotherapy and surgery, the same percentage received radiotherapy and chemotherapy, and $7.1 \%$ received a combination of these three types of treatment. Radiation treatment consisted of exclusive external radiotherapy in 28 of the cases $(28.6 \%)$. In the remaining 70 cases, external radiotherapy was combined with intracavitary brachytherapy. Surgery consisted of 27 simple hysterectomies, 13 radical hysterectomies and 9 low anterior resections (Table 3). Considering the time gone from the conclusion of the radiation treatment to the moment of data collection, the minimum time was 3 months and the maximum 58 months, with an average of $15.4 \pm 10.6$ months; $25.5 \%$ of patients had concluded the treatment less than 6 months before, 25.5.\% 6-12 months before, and 49\% more than 12 months before. Regarding present clinical situation, most women were free from active cancer at the time of the
Table 1 Socio-demographic data

\begin{tabular}{|c|c|c|c|c|c|}
\hline & \multicolumn{2}{|c|}{ Study group $(n=98)$} & \multicolumn{2}{|c|}{ Control group $(n=101)$} & \multirow[t]{2}{*}{$p$} \\
\hline & $N$ & $\%$ & $N$ & $\%$ & \\
\hline \multicolumn{6}{|l|}{ Age } \\
\hline$\leq 40$ & 12 & 12.3 & 12 & 11.9 & n.s. \\
\hline $41-60$ & 35 & 35.7 & 47 & 46.5 & n.s. \\
\hline$>60$ & 51 & 52.0 & 42 & 41.6 & n.s \\
\hline \multicolumn{6}{|l|}{ Education } \\
\hline Primary education & 66 & 67.3 & 63 & 62.4 & n.s. \\
\hline Secondary education & 24 & 24.5 & 28 & 27.7 & n.s. \\
\hline Higher education & 8 & 8.2 & 10 & 9.9 & n.s. \\
\hline \multicolumn{6}{|c|}{ Present employment situation } \\
\hline Paid employment & 24 & 24.5 & 35 & 34.7 & n.s. \\
\hline Non-paid employment & 19 & 19.4 & 21 & 20.8 & n.s. \\
\hline Unemployed & 10 & 10.2 & 6 & 5.9 & n.s. \\
\hline Retired & 45 & 45.9 & 39 & 38.6 & n.s. \\
\hline \multicolumn{6}{|l|}{ Place of residence } \\
\hline Rural areas & 68 & 69.4 & 80 & 79.2 & n.s. \\
\hline Urban areas & 30 & 30.6 & 21 & 20.8 & n.s. \\
\hline
\end{tabular}


study $(91.8 \%)$. From the women included in the control group, 35 (34.7\%) were pre-menopausal and 66 (65.3\%) were menopausal. Of the latter, only $3(4.5 \%)$ were using hormone replacement therapy.

Table 2 Tumor distribution according to stage

\begin{tabular}{llc}
\hline Tumor localization & Stage & Number of cases \\
\hline Cervical cancer $(n=48)$ & IB2 & 7 \\
& IIA & 3 \\
& IIB & 13 \\
& IIIA & 6 \\
Endometrial cancer $(n=34)$ & IIIB & 15 \\
& IVA & 4 \\
& IA & 2 \\
& IC & 9 \\
& IIA & 5 \\
Rectal cancer $(n=13)$ & IIB & 7 \\
& IIIA & 2 \\
& IIIB & 3 \\
& IIIC & 6 \\
Anal canal cancer $(n=3)$ & IIA & 2 \\
& IIIA & 4 \\
& IIIB & 6 \\
& IIIC & 1 \\
& I & 1 \\
& II & 2 \\
\hline
\end{tabular}

Measures

\section{Socio-demographic data}

Age, education, present employment situation, place of residence and parity.

\section{Clinical data}

Study participants were asked about their present hormonal status, as well as about their hormonal status prior to treatment and about their use of hormone replacement therapy. The presence of undesirable physical symptoms in the preceding month was assessed through a self-reported questionnaire that included 15 items: pain, nausea, fatigue, loss of appetite, weight loss, loss of strength, vaginal bleeding, vaginal discharge, diarrhoea, constipation, haematuria, dysuria, rectal bleeding, skin erythema and insomnia/sleep disorders. Clinical data, namely tumor characteristics and radiotherapy techniques used, were complemented by consultation of clinical records.

\section{Sexual function and satisfaction}

Sexual function and satisfaction were assessed by the Female Sexual Function Index (FSFI: Rosen et al. [12] Portuguese Version by Hentshel et al. [13]), composed of 19 items assessing sexual activity during the preceding 4 weeks. This index measures six dimensions of female

Table 3 Type of treatment

\begin{tabular}{|c|c|c|}
\hline & Type of treatment & $\begin{array}{l}\text { Number } \\
\text { of cases }\end{array}$ \\
\hline \multirow{4}{*}{$\begin{array}{l}\text { Cervical cancer } \\
\quad(\mathrm{n}=48)\end{array}$} & $\mathrm{S}[\mathrm{RH}]+\mathrm{EBRT}(50.4 \mathrm{~Gy})+\mathrm{HDR}$ BT (28 Gy) & 12 \\
\hline & EBRT (50.4-64.8 Gy) + CT[cisplatin] & 10 \\
\hline & EBRT (50.4-64.8 Gy) + CT[cisplatin] + HDR BT (14-28 Gy) & 25 \\
\hline & EBRT $(59.4$ Gy $)$ & 1 \\
\hline \multirow[t]{4}{*}{$\begin{array}{l}\text { Endometrial cancer } \\
\qquad(n=34)\end{array}$} & $\begin{array}{l}\text { S[TAH-BSO-12; TAH-BSO with pelvic lymphadenectomy-12; RH-1] + HDR BT } \\
(28 \text { Gy) + EBRT (50.4 Gy) }\end{array}$ & 25 \\
\hline & $\begin{array}{l}\text { S[TAH-BSO with pelvic lymphadenectomy] + HDR BT }(28 \text { Gy })+\text { EBRT } \\
(50.4 \text { Gy })+\text { CT[doxorubicin and cisplatin] }\end{array}$ & 3 \\
\hline & EBRT (40 Gy) + HDR BT (35 Gy) & 5 \\
\hline & EBRT $(68.4$ Gy) + CT[doxorubicin and cisplatin] & 1 \\
\hline \multirow{5}{*}{$\begin{array}{l}\text { Rectal cancer } \\
\quad(n=13)\end{array}$} & $\mathrm{S}[\mathrm{LAR}]+\mathrm{EBRT}(50.4 \mathrm{~Gy})$ & 4 \\
\hline & $\mathrm{S}[\mathrm{LAR}]+$ EBRT (50.4 Gy) + CT[5-Fluorouracil] & 4 \\
\hline & $\operatorname{EBRT}(50.4 \mathrm{~Gy})+\mathrm{S}[\mathrm{LAR}]$ & 1 \\
\hline & EBRT (50.4 Gy) + CT[5-Fluorouracil] & 3 \\
\hline & EBRT (66.6 Gy) & 1 \\
\hline $\begin{array}{l}\text { Anal canal cancer } \\
(n=3)\end{array}$ & EBRT (64.8 Gy) + CT[5-Fluorouracil and mitomycin-C] & 3 \\
\hline
\end{tabular}

$S$ surgery, EBRT external-beam radiation therapy, $H D R B T$ hight-dose rate brachytherapy, $C T$ chemotherapy, $R H$ radical hysterectomy, $T A H$ total abdominal hysterectomy, $B S O$ bilateral salpingo-oophorectomy 
sexual function (desire-2 items; arousal -4 items; lubrication-4 items; orgasm-3 items; satisfaction-3 items; pain-3 items) and it also provides a score for female sexual function as a whole. Response options were organized according to a Likert-type scale of $1-5$ for items 1,2 , 15 and 16. In the remaining items, response options ranged from 0 to 5 , since they included the option 'no sexual activity'. The score of each domain was obtained by adding up individual scores, then multiplying the obtained score by the corresponding factor. The total score of the scale was obtained by adding up the scores of each domain. The level of total sexual function can range between 2 and 36, low scores meaning the existence of multiple problems related to sexual function, and high scores representing good sexual function. In order to complete the assessment of the sexual component, three further questions were included: 'Reasons for not having sexual activity'; 'Has your treating doctor ever addressed the topic of sexuality?'; 'Have you ever talked to your treating doctor about your sex life on your own initiative?'

\section{Depression, anxiety and stress}

Depression, anxiety and stress were assessed by the Depression Anxiety Stress Scale-21 (DASS-21: Lovibond and Lovibond [14]—Portuguese Version by Pais-Ribeiro et al. [15]). The original version of this scale aimed at empirically assessing anxiety, depression and stress (including the least discriminative items from the latter two dimensions) through 42 items. A short version of this 42-item scale was created with 21 items, subdivided into 7 items by dimension. Each item consisted of a statement connected to negative emotional symptoms. Interviewees were asked to answer whether the statement applied to them in the preceding week. For each statement, four answering options were presented using a Likert-type scale with the following phrases: 'did not apply to me at all'; 'sometimes applied to me'; 'often applied to me'; and 'most always applied to me'. The results of each scale were obtained by adding up the results of the seven items, ranging between 0 and 21 . The highest scores of each scale indicated the most negative emotional states.

\section{Satisfaction with social support}

This dimension was assessed by the Satisfaction with Social Support Scale (ESSS: Ribeiro [16]). This scale measures the perception of the existence of social support and is composed of 15 statements for self-completion. Interviwees should tick according to the extent to which they agreed with the statement on a five-point Likert-type scale: 'strongly agree', 'mostly agree', 'neither agree nor disagree', 'mostly disagree', and 'strongly disagree'. Items 4 ,
$5,9,10,11,12,13,14$ and 15 were reverse scored. Questions were divided into four dimensions. The first dimension-'Satisfaction with friends'-measured satisfaction with friendships and included five items $(3,12,13$, 14 and 15). The second dimension-'Intimacy'-measured the perception of the existence of social intimacy support and included four items (1, 4, 5 and 6). The third dimension-'Satisfaction with family'-measured satisfaction with family social support and included three items $(9,10$ and 11). The fourth dimension-'Social activity'-measured satisfaction with social activities and included three items (2, 7 and 8). The score for each dimension was obtained by the sum of the items in each of the dimensions. The total score of the scale was obtained by the sum of all items. The total score of the scale can range between 15 and 75, where the highest scores indicated a higher perception of social support.

\section{Relationship satisfaction}

Relationship satisfaction was assessed by the Index of Marital Satisfaction (IMS: Hudson [17]—Portuguese Version by Pereira et al. [18]) aiming to measure women perception of their couple relationship. This tool is composed of 25 items which assess the degree, severity and magnitude of relationship problems. Response options ranged on a 7-point Likert-type scale from: $1=$ 'never', $2=$ 'very rarely', $3=$ 'rarely' $\quad 4=$ 'sometimes', $5=$ 'often', $6=$ 'nearly always' and $7=$ "always'. Questions 1, 3, 5, 8, 9, 11, 13, 16, 17, 19, 20 and 23 were reverse scored. The final score was the result of the sum of all items, to which 25 was subtracted, multiplied by 100 and divided by 150 . The final score ranges between 0 and 100 , highest scores indicating greater severity of relationship problems.

\section{Statistical analysis}

The variables of age, education, employment situation, place of residence, physical morbidity, psychological morbidity, sexual function, satisfaction with social support and couple relationship were compared between the study group and the control group using Chi-square and MannWhitney tests.

Considering the study group only, we compared the dimensions of sexual function according to the time lapse since the end of the radiation treatment ( $<6$ months, between 6 and 12 months, $>12$ months), therapeutic modality (exclusive radiotherapy, radiotherapy-surgery, radiotherapy-chemotherapy and radiotherapy-chemotherapy-surgery), age ( $\leq 40,41-60$ and $>60$ years of age), hormonal status before treatment, use of hormone replacement therapy, presence of active cancer, tumor 
location (uterine vs. intestinal) and type of hysterectomy (simple vs. radical). Non-parametric tests were used (Mann-Whitney or Kruskal-Wallis tests).

In the study group, associations were found between the variables of total radiation dose, physical morbidity (considering the number of symptoms reported by each woman), DASS score, ESSS score, IMS score, and FSFI score, using Spearman's rank correlation. The correlation between sexual function and total radiation dose was assessed by separately considering women who received exclusive external pelvic radiotherapy and women who received both external pelvic radiotherapy and intracavitary brachytherapy, since the biological effects of the same dose when exclusively administered by external radiation and when administered through combined external and intracavitary irradiation are different.

The data obtained were encoded and processed through the Statistical Packages for Social Sciences softwareversion 16 (SPSS-16 $\left.{ }^{\circledR}\right)$.

\section{Results}

The occurrence of all the physical symptoms enquired about was higher in the group of women who received radiation, with a mean number of symptoms reported by each woman of $3.2 \pm 2.2$ versus $2.0 \pm 2.1(p<0.001)$. Nevertheless, statistically significant differences were only found in the variables of 'Fatigue' (59.2 vs. 24.8\%, $p<0$. 001), 'Lack of strength' (41.8 vs. 19.8\%, $p=0.001)$, 'Vaginal discharge' (17.3 vs. 6.9\%, $p=0.024)$, 'Diarrhoea' (23.5 vs. $11.9 \%, p=0.032)$ and 'Skin erythema' (9.2 vs. $2.0 \%, p=0.026$ ) (Table 4). Concerning psychological morbidity, the study group presented higher scores in all domains of DASS and in the total scale, indicating more negative emotional states. However, this difference was only statistically significant in the dimension of 'Stress' (6.1 \pm 5.2 vs. $4.0 \pm 3.2, p=0$. 012). The score for the global FSFI and for all domains of sexual function was significantly lower in the study group, with the exception of the 'Satisfaction' domain. Bearing in mind that the score of the 'Desire' domain can range between a minimum of 1.2 and a maximum of 6 , we found that this domain of sexual function was the most highly compromised. The two groups also differ in terms of sexual abstinence in the preceding 4 weeks (60.2 vs. $44.6 \%$, $p=0.033$ ). In the study group, the most common reasons for being sexually abstinent were morbidity resulting from cancer diagnosis and treatment $(66.1 \%)$ and illness or physical limitation of the sexual partner that made intercourse impossible, uncomfortable or embarrassing (11.9\%). Controls reported low sexual interest $(37.0 \%)$ and illness or physical limitation of the sexual partner $(21.7 \%)$ as the most common reasons for sexual abstinence. Satisfaction with friends and family and global satisfaction with social support were significantly lower in the group of women who received pelvic radiotherapy. Relationship satisfaction did not present statistically significant differences between both groups $(20.8 \pm 18.5$ vs. $19.9 \pm 22.2$, $p=$ n.s.) (Table 5).

Considering the study group only, and sexual function according to the time lapse after the end of the radiation treatment, lower scores were obtained in the six domains of sexual function when there was a longer period of time since the end of the treatment. Nevertheless, these differences did not have statistical significance. Physical and psychological morbidity also did not differ according to the time lapse since the end of the radiation treatment. Women who received exclusive radiotherapy presented lower scores for sexual function in terms of the global scale $(p=0.042)$, as well as for the domains of 'Desire' $(p=0.020)$, 'Arousal' $(p=0.043)$, 'Lubrication' $(p=0.029)$, 'Orgasm' $(p=$ $0.024)$ and 'Pain' ( $p=0.029)$, when compared to women who received combined therapeutic modalities. No differences were found regarding simple versus radical hysterectomy. Women aged 40 or over presented higher scores in all domains of the FSFI, but only the scores for the items 'Desire' ( $p<0.001)$, 'Arousal' ( $p=0.019)$, 'Lubrication' $(p=0.035)$ and for the global scale $(p=0.031)$ were statistically significant. Women who were pre-menopausal before the radiation treatment had higher scores in all dimensions of sexual function (Desire: $2.5 \pm 1.2$ vs. $1.5 \pm 0.9, p<0$. 001; Arousal: $1.8 \pm 1.9$ vs. $0.7 \pm 1.4$, $p=0.002$; Lubrication: $2.0 \pm 2.1$ vs. $0.8 \pm 1.5, p=$ 0.003 ; Orgasm: $1.7 \pm 2.0$ vs. $0.8 \pm 1.5, p=0.006$; Satisfaction: $2.7 \pm 2.1$ vs. $2.0 \pm 1,7, p=0.144$; Pain: $1.8 \pm$ 2.0 vs. $0.9 \pm 1.8, p=0.007$; Total: $12.4 \pm 10.1$ vs. $6.7 \pm$ $7.7, p=0.006$ ). No statistically significant differences were found regarding the use of hormone therapy, presence of active cancer or tumor location.

The association between the sexual function and total radiation dose was not statistically significant. The variables Depression-Desire $(r=-0.132 ; p=0.04)$, DepressionArousal $(r=-0.247 ; p=0.015)$, Depression-Lubrication $(r=-0.257 ; p=0.012)$, Depression-Orgasm $(r=-0.231$; $p=0.023)$, Depression-Pain $(r=-0.218 ; p=0.033)$, DASS Total-Arousal $(r=-0.225 ; p=0.028)$ and DASS Total-Lubrication $\quad(r=-0.233 ; \quad p=0.022) \quad$ correlated significantly.

Out of the 98 women who composed the study group, only $25.5 \%$ mentioned that their doctors questioned them about their sexuality, while $17.3 \%$ reported having addressed the topic of sexuality with their treating doctor on their own initiative. 
Table 4 Physical morbidity

\begin{tabular}{|c|c|c|c|c|c|}
\hline & \multicolumn{2}{|c|}{ Study group $(n=98)$} & \multicolumn{2}{|c|}{ Control group $(n=101)$} & \multirow[t]{2}{*}{$p$} \\
\hline & $\mathrm{N}$ & $\%$ & $\mathrm{~N}$ & $\%$ & \\
\hline Pain & 36 & 36.7 & 27 & 26.7 & n.s. \\
\hline Nausea & 22 & 22.4 & 15 & 14.9 & n.s. \\
\hline Fatigue & 58 & 59.2 & 25 & 24.8 & $<0.001$ \\
\hline Loss of appetite & 17 & 17.3 & 10 & 9.9 & n.s. \\
\hline Weight loss & 15 & 15.3 & 9 & 8.9 & n.s \\
\hline Loss of strength & 41 & 41.8 & 20 & 19.8 & 0.001 \\
\hline Vaginal Bleeding & 4 & 4.1 & 2 & 2.0 & n.s. \\
\hline Vaginal discharge & 17 & 17.3 & 7 & 6.9 & 0.024 \\
\hline Diarrhoea & 23 & 23.5 & 12 & 11.9 & 0.032 \\
\hline Constipation & 22 & 22.4 & 20 & 19.8 & n.s. \\
\hline Rectal bleeding & 9 & 9.2 & 3 & 3.0 & n.s. \\
\hline Dysuria & 11 & 11.2 & 10 & 9.9 & n.s. \\
\hline Insomnia/sleep disorders & 33 & 33.7 & 25 & 24.8 & n.s. \\
\hline Skin erythema & 9 & 9.2 & 2 & 2.0 & 0.026 \\
\hline Haematuria & 2 & 2.0 & 1 & 1.0 & n.s. \\
\hline
\end{tabular}

Table 5 DASS, FSFI, ESSS and IMS scores

\begin{tabular}{|c|c|c|c|c|c|}
\hline & \multicolumn{2}{|c|}{ Study group $(n=98)$} & \multicolumn{2}{|c|}{ Control group $(n=101)$} & \multirow[t]{2}{*}{$p$} \\
\hline & Mean & SD & Mean & SD & \\
\hline \multicolumn{6}{|c|}{ Depression anxiety stress scale } \\
\hline Anxiety & 3.5 & 3.9 & 3.3 & 2.5 & n.s \\
\hline Depression & 4.4 & 5.0 & 2.2 & 1.9 & n.s. \\
\hline Stress & 6.1 & 5.2 & 4.0 & 3.2 & 0.012 \\
\hline Total & 13.9 & 13.0 & 9.5 & 4.5 & n.s. \\
\hline \multicolumn{6}{|l|}{ Female sexual function index } \\
\hline Desire & 1.8 & 1.1 & 2.4 & 1.6 & 0.004 \\
\hline Arousal & 1.0 & 1.6 & 1.7 & 2.2 & 0.033 \\
\hline Lubrication & 1.2 & 1.8 & 2.0 & 2.4 & 0.024 \\
\hline Orgasm & 1.1 & 1.7 & 2.1 & 2.5 & 0.004 \\
\hline Satisfaction & 2.3 & 1.8 & 2.8 & 2.1 & n.s. \\
\hline Pain & 1.2 & 1.9 & 2.3 & 2.7 & 0.005 \\
\hline Total & 8.5 & 8.9 & 13.5 & 13.0 & 0.049 \\
\hline \multicolumn{6}{|c|}{ Satisfaction with social support scale } \\
\hline Satisfaction with friends & 19.8 & 5.1 & 22.2 & 4.1 & $<0.001$ \\
\hline Intimacy & 15.0 & 3.9 & 16.0 & 3.8 & n.s. \\
\hline Satisfaction with family & 12.4 & 3.4 & 13.4 & 2.8 & 0.011 \\
\hline Social activity & 10.0 & 3.9 & 10.1 & 3.7 & n.s. \\
\hline Total & 57.2 & 12.9 & 62.2 & 11.0 & 0.005 \\
\hline \multicolumn{6}{|l|}{ Index of marital satisfaction } \\
\hline Relationship satisfaction & 20.8 & 18.5 & 19.9 & 22.2 & n.s. \\
\hline
\end{tabular}

\section{Discussion}

Treatment of pelvic malignancies with radiotherapy affects women's sexual function [3-11, 19, 20]. In their critical review of 23 patient-rated quality of life studies of longterm survivors of cervical cancer, Vistad et al. [4] mentioned a significant reduction of sexual desire and lubrication and higher occurrence of dyspareunia in women submitted to pelvic radiotherapy. Marijnen et al. [20] concluded that the use of preoperative radiotherapy in rectal cancer patients led to more sexual dysfunction. Similarly, the results obtained in this study revealed lower 
sexual function scores in women who received pelvic irradiation compared to the control group, sexual desire being the most affected domain. Jensen et al. [8] reported that approximately $85 \%$ of women had low or no sexual interest after radiotherapy for cervical cancer. Desire disorders can be explained by the deficit of sexual hormones caused by ovarian irradiation, depression, pain, asthenia and sleep disorders $[3,6,21]$. The absence of statistically significant differences in the item of 'Satisfaction' implies that women tend to adapt sexual intercourse in order to make it satisfactory. Even in the presence of sexual problems, feeling and giving pleasure are possible [22].

The emergence of sexual dysfunctions after radiation exposure of the female pelvic area is multifactorial, arising as a result of physiological, psychological and sociological difficulties $[3,7]$. The results of this study showed higher prevalence of physical and psychological symptoms in women exposed to pelvic irradiation, corroborating Cull et al. [7]. Fatigue and loss of strength were the most common physical symptoms. Concerning social support, we found lower perception of social support in the study group, which can be explained by the relational impoverishment secondary to physical, emotional and sexual complaints reported not only by the patients, but also by their relatives and friends [23].

Sexual function is also highly dependent on the quality of the couple relationship [7, 11, 24]. When faced with the cancer and its treatment, partners may drift away by believing that cancer is contagious, that radiation can be transmitted, by fear of hurting women or even due to physical and psychosocial disorders [3, 5, 7, 24]. Our results do not reveal a significant increase in couple relationship problems in the group of women exposed to pelvic radiotherapy. Nevertheless, Flay et al. [5] reported that $18 \%$ of women treated with radiotherapy for cervical cancer have major relationship problems by the 14-week follow-up.

As in this study, previous research showed that sexual problems, physical and psychological morbidity do not necessarily improve over time and can even intensify [3, 4, $8,11]$. This evolution is connected to late effects of radiation, which tend to appear 6-12 months after the end of treatment and which are often irreversible [10]. Tiredness, anorexia, depression and sleep disorders also tend to persist over long periods of time [6, 7]. Moreover, even after successful treatment, patients keep memories of the negative feelings experienced throughout radiotherapy sessions, show fear of cancer recurrence, and could even develop post-traumatic stress disorder symptoms [6, 7, 25]. They feel more vulnerable because hospital appointments become increasingly less frequent and social support tends to decrease over time $[6,23]$. Finally, in the absence of adequate clinical intervention, sexual problems tend to be perpetuated; in fact, couples seek to minimize the anxiety associated with sexual activity by avoiding any kind of intimacy [26].

The combination of pelvic radiotherapy with other therapeutic modalities is generally associated with a deteriorated sex life, as it implies more complex side effects, more trips to the hospital, longer hospital stays, greater socio-economic implications and more psychological problems $[4,5,9,27]$. The low scores obtained in our sample by women who received exclusive radiotherapy may be explained by a sample bias; the small number of women in this subgroup makes it difficult to draw definitive conclusions about the sexual effects of different treatment modalities.

Regarding women's age, our results corroborate Pignon et al. [28] that reported a higher number of immediate and late sexual dysfunction in elderly people after radiotherapy, which was attributed to physiological alterations typical for their age and to co-morbidities. On the contrary, Burwell et al. [29] and Miller et al. [19] mentioned that sexual problems are particularly serious in younger and premenopausal women with cancer diagnosis, since these are usually more concerned about their body image, suffer greater psychological stress, give greater importance to sex, are more vulnerable to alterations in ovarian function and subjected to more aggressive treatments.

Hormone replacement therapy is a hormonal treatment administered at menopause with the purpose of regaining hormonal balance and avoiding the consequences of ovarian failure and endogenous hormone deprivation. Donovan et al. [11], who specifically studied the sexual function of women with cervical cancer submitted to pelvic radiotherapy, reported more sexual interest when hormone therapy was administered. In the present study, no statistically significant differences were found in sexual function regarding the use or non-use of hormone replacement therapy.

The persistence of the disease or its recurrence generally implies more treatments and, consequently, greater physical and psychological suffering, as well as lower scores for sexual function [19]. Nevertheless, this was not confirmed by results. The fact that only eight patients had an active cancer at the moment of data collection can explain the absence of statistically significant differences between women with and without an active cancer.

Consequences deriving from cancer depend on the type of tumor and on the emotional/sexual significance of the affected organ [3]. In the present study, no statistically significant differences were found in sexual function regarding tumor location. Estapé [30] mentions that cancer and its treatment repercussions on female sexuality are more severe when genital organs are directly affected. However, the treatment for intestinal cancer often creates alterations in the intestinal transit and abdominal 
discomfort, which restricts sexual contact [3]. Lange et al. [9] found that the presence of a stoma was a risk factor for sexual dysfunction, because body image is deteriorated and anxiety toward odor appears, as well as the fear of displacing the bag and releasing faeces [3].

Radiation does not affect tumor cells only; rather, it also affects adjacent cells, thereby causing adverse effects, which can be more or less frequent and serious according to the administered dose $[5,28]$. Nevertheless, the results obtained did not prove any significant correlation between sexual function and total radiation dose.

Emotional disorders are common after the diagnosis and treatment of malignant diseases [3, 6, 7]. The present study revealed a statistically significant correlation between the following variables: Depression-Desire, DepressionArousal, Depression-Lubrication, Depression-Orgasm; Depression-Pain, DASS Total-Arousal and DASS TotalLubrication. Cull et al. [7] also found a correlation between psychological and sexual outcome and Chochinov et al. [31] described depression as the main psychological cause of decreased libido in patients with cancer. This relation can be explained by the action of dopamine and other neurotransmitters on the sexual hormones. Merely the symptoms of cancer can leave women exhausted and unable to engage in any sexual activity. The prescription of antidepressants can also have an impact on sexuality by aggravating the reduced sexual desire. Similarly, changes in sexuality and femininity can lead to the development of depressive symptoms [3, 6].

As results have shown, a reduced number of women suffering from sexual dysfunction enquire about sexuality or look for professional help for this kind of problems; in the same way, health professionals also seem to find it difficult to address the topic of sexuality. In a study carried out by Lindau et al. [32], 62\% of survivors of genital cancer had never spoken to their doctors about the effects of treatments on sexuality. Lack of privacy in hospital environment, feeling guilty about sexual desire, and inhibition due to young age or to the gender of the interlocutor can prevent discussions on the topic $[3,4]$. Therefore, there is an obligation for clinicians to regularly address this topic [8]. Nevertheless, despite major breakthroughs in this area, the impact of cancer on sexual function tends not to be given as much importance as its impact on other life spheres of patients, since many doctors believe that only young and healthy women are concerned with issues related to sexuality [33]. Another reason for the inability to communicate on sexuality is the lack of training and of aptitude by some clinicians, which makes them uncomfortable in addressing the topic of sex. Other clinicians believe that if sexuality is a problem for patients, they will address it themselves, or even that this kind of dialogue is not an area of their competence or is too time-consuming [7].
This is one of the first clinical studies to assess the sexual function as a multidimensional variable through validated questionnaires, including the physical, psychological and relationship factors that contribute to the development of female sexual dysfunction. However, there are some limitations that should be noted: it is a crosssectional study conducted after the end of radiation treatment so exist the possibility that differences after the end of the treatment reflect pre-treatment differences; it does not assess the presence of sexual problems in the partner; the number of women who received radiotherapy alone or with persistent or recurrent disease is small, which prevents the establishment of definitive conclusions regarding those subgroups. Vaginal late effects, such as shortening and stenosis, were insufficiently recorded due to lack of specific instruments validated for the Portuguese language. Thus, it would be of great importance to continue research on this subject through longitudinal studies, as well as to evaluate the impact on the partner and the family, in order to clarify the results obtained in this study and to contribute to a more solid basis for intervention by health professionals.

In conclusion, the results of our study emphasize that women treated with pelvic radiotherapy are at risk for physical, psychological and sexual problems, which tend to persist several months to years after the end of treatment. Despite the fact that treatment of sexual dysfunctions requests a multidisciplinary approach, physicians can do much for the welfare of patients with simple measures that should be encouraged, such as asking and informing. We believe that all oncologists must be prepared to give information about the anatomy and physiology of sexual response and about possible consequences of radiation on sexuality and body image, demystify false beliefs about sexuality, cancer and radiotherapy, provide emotional support, minimize extragenital effects of radiotherapy, and diagnose and recommend expert help for sexual problems after radiotherapy.

Conflict of interest None.

\section{References}

1. Annon J (1976) Behavioral treatment of sexual problems. Harper \& Row Publishers, Hagerstown

2. Auchincloss SS (1998) Sexual dysfunction in cancer patients: issues in evaluation and treatment. In: Holland JC, Rowland JH (eds) Handbook of psychooncology: psychological care of patient with cancer. Oxford University Press, New York, pp 383-413

3. Baucom DH, Porter LS, Kirby JS, Gremore TM, Keefe FJ (2005) Psychosocial issues confronting young women with breast cancer. Breast Dis 23:103-113

4. Bergmark K, Avall-Lundqvist E, Dickman PW, Henningsohn L, Steineck G (1999) Vaginal changes and sexuality in women with a history of cervical cancer. N Engl J Med 340:1383-1389 
5. Boyle P, Ferlay J (2005) Cancer incidence and mortality in Europe, 2004. Ann Oncol 16:481-488

6. Burwell SR, Case D, Kaelin C, Avis NE (2006) Sexual problems in younger women after breast cancer surgery. J Clin Oncol 24:2815-2821

7. Chochinov H (2001) Depression in cancer patients. Lancet Oncol 2:449-505

8. Cull A, Cowie VJ, Farquharson DIM, Livinstone JRB, Smart GE, Elton RA (1993) Early satge cervical cancer: psychosocial and sexual outcomes of treatment. Br J Cancer 68:1216-1220

9. Donovan KA, Taliaferro LA, Alvarez EM, Jacobsen PB, Roetzheim RG, Wenham RM (2007) Sexual health in women treated for cervical cancer: characteristics and correlates. Gynecol Oncol 104:428-434

10. Estapé T, Estapé J, Munõz M, Firvida J, Vinõlas N (1993) Sexual dysfunctions among cancer patients. Eur J Cancer 6:213

11. Flay LD, Matthews JHL (1995) The effects of radiotherapy and surgery on the sexual function of women treated for cervical cancer. Int J Radiat Oncol Biol Phys 31:399-404

12. Hawighorst-Knapstein S, Fusshoeller C, Franz C, Trautmann K, Schmidt M, Pilch H et al (2004) The Impact of treatment for genital cancer on quality of life and body image-results of a prospective longitudinal 10-year study. Gynecol Oncol 94:398-403

13. Hentschel H, Alberton D, Capp E, Goldim J, Passos E (2007) Validação do Female Sexual Function Index para o uso em Língua Portuguesa (Validation of the female sexual function index for use in Portuguese). Rev HCPA 27:11-14

14. Hudson W (1992) The WALMYR assessment scales scoring manual. Walmyr Publishing, Tempe

15. Hughes MK (2008) Alterations of sexual function in women with cancer. Semin Oncol Nurs 24:91-101

16. Jemal A, Siegel R, Ward E, Hao Y, Xu J, Thum MJ (2009) Cancer Statistics, 2009. CA Cancer J Clin 54:225-249

17. Jensen PT, Mogens G, Klee MC, Thranov I, Petersen MA, Machin D (2003) Longitudinal study of sexual function and vaginal changes after radiotherapy for cervical cancer. Int $\mathbf{J}$ Radiat Oncol Biol Phys 56:937-949

18. Lange MM, Marijnen CAM, Maas CP, Putter H, Rutten HJ, Stiggelbout AM et al (2009) Risk factors for sexual dysfunction after rectal cancer treatment. Eur J Cancer 45:1578-1588

19. Lewis F (1989) Strengthening family Supports. Cancer and family. Cancer 13:754-759

20. Lindau ST, Gravrilova N, Anderson D (2007) Sexual morbidity in very long term survivors of vaginal and cervical cancer: a comparison to national norms. Gynecol Oncol 106:413-418

21. Lovibond P, Lovibond S (1995) The structure of negative emotional states: comparison of the depression anxiety scales (DASS) with the beck depression and anxiety inventories. Behav Res Ther 33:335-343

22. Marijnen CA, van de Velde CJ, Putter H, van den Brink M, Maas $\mathrm{CP}$, Martijn $\mathrm{H}$ et al (2005) Impact of short-term preoperative radiotherapy on health-related quality of life and sexual functioning in primary rectal cancer: report of a multicenter randomized trial. J Clin Oncol 23:1847-1858

23. McCoy NL (1994) Survey research on the menopause and women's sexuality. In: Berg G, Hammer M (eds) The modern management of the menopause: a perspective for the 21 st century. Parthenon, Lancaster, pp 581-588

24. Merck Manuals (2005) Sexual dysfunction in women: gynecology and obstetrics. http://www.merck.com/mmpe/print/sec18/ ch251/ch251a.html (Accessed 4th September, 2009)

25. Miller BE, Pittman B, Case D, McQuellon RP (2002) Quality of life after treatment for gynecologic malignancies: a pilot study in an outpatient clinic. Gynecol Oncol 87:178-184

26. Pais-Ribeiro J, Honrado A, Leal I (2004) Contribuição para o estudo da adaptação portuguesa das escalas de Ansiedade, Depressão e Stress de 21 itens de Lovibond e Lovibond (Contribution to the study of the Portuguese adaptation of the 21-item Anxiety, depression and stress scale by Lovibond and Lovibond). Psicologia Saúde Doenças 5:229-239

27. Perdrizet-Chevallier C, Reich M, Lesur A (2008) Dépression et anxiété chez les femmes souffrant de cancers gynécologiques (Depression and anxiety in women with gynecological cancer). Ann Med Psychol 166:292-296

28. Pereira M, Ramalho V, Dias P (2000) Inventário de Satisfação Marital [Index of marital satisfaction]. Minho University, Braga

29. Pignon T, Horiot JC, Bolla M, Poppel H, Bartelink H, Roelofsen F et al (1997) Age is not a limiting factor for radical radiotherapy in pelvic malignancies. Radiother Oncol 42:107-120

30. Ribeiro JLP (1999) Escala de Satisfação com o Suporte Social (Social support satisfaction scale). Análise Psicológica 3:547-558

31. Rosen R, Brown C, Heiman J, Leiblum S, Fergunson D, Agostino $\mathrm{R}$ (2007) The female sexual function index (FSFI): a multidimensional self-report instrument for the assessment of female sexual function. J Sex Marital Ther 26:191-208

32. Schover L, Montague D, Lakin M (1997) Sexual Problems. In: Devita VT, Hellman S, Rosenberg SA (eds) Cancer: principles and practices of oncology, 5th edn. Lippincott-Raven, Philadelphia, pp 2857-2871

33. Vistad I, Fossa SD, Dahl AA (2006) A critical review of patientrated quality of life studies of long-term survivors of cervical cancer. Gynecol Oncol 102:563-572 